\title{
Study of Problem Solving Following Herbert Simon
}

\author{
Yulin Qin ${ }^{1,2}$ and Ning Zhong ${ }^{1,3}$ \\ 1 The International WIC Institute, Beijing University of Technology, China \\ 2 Dept. of Psychology, Carnegie Mellon University, USA \\ 3 Dept. of Life Science and Informatics, Maebashi Institute of Technology, Japan \\ yulinqin@gmail.com, zhong@maebashi-it.ac.jp
}

Herbert Simon (1916.6.15 - 2001.2.9) was one of the greatest pioneers in cognitive science and artificial intelligence, as well as in behavior economics and many other fields. Problem solving was his core work in artificial intelligence and cognitive psychology. He and Newell first postulated a general and systematic framework of human (and machine) problem solving as iteratively applying operators to transform the state of the problem from the starting state in problem state space to eventually achieve the goal state. Heuristic problem solving includes two basic components: heuristic searching (such as means-ends analysis) and heuristic rules (used to change the problem states). And then, he extended this framework in two dimensions. One is applying this framework to creative learning and scientific discovery (both were thought as specific ill-structured problem solving tasks); the other is to elaborate this general framework with more detailed models in memory (such as chunk structure in short term memory) and the knowledge (and problem) representations, including the knowledge structure difference between experts and naives, diagrammatic representation and mental imagery. To meet the challenge of Web intelligence and to pioneer the effective and efficient ways of information processing at Web scale, as the first step, we would learn this process from human brain, one of the greatest webs, based on Simon and Newell's framework in problem solving. We have found that, even in the basic application of heuristic rules, the processes are distributed in several major parts of brain and with certain areas for the communications across these networks. We have checked the brain activations in regard to working memory and mental imagery in problem solving. We have also found the evidences supporting the hypothesis that the scientific discovery is a specific problem solving from neural activations that central brain areas activated in scientific discovery overlapping with the areas in general problem solving tasks. These findings offer strong clues for how to solve problems at Web scale. 